PROCEEDINGS OF THE

AMERICAN MATHEMATICAL SOCIETY

Volume 135, Number 11, November 2007, Pages 3695-3702

S 0002-9939(07)08951-4

Article electronically published on August 7, 2007

\title{
DOES NEGATIVE TYPE CHARACTERIZE THE ROUND SPHERE?
}

\author{
SIMON LYNGBY KOKKENDORFF
}

(Communicated by Jon G. Wolfson)

\begin{abstract}
We discuss the measure-theoretic metric invariants extent, mean distance and symmetry ratio and their relation to the concept of negative type of a metric space. A conjecture stating that a compact Riemannian manifold with symmetry ratio 1 must be a round sphere was put forward by the author in 2004. We resolve this conjecture in the class of Riemannian symmetric spaces by showing that a Riemannian manifold with symmetry ratio 1 must be of negative type and that the only compact Riemannian symmetric spaces of negative type are the round spheres.
\end{abstract}

\section{INTRODUCTION}

We will discuss the concept of negative type of a metric space, focusing on the relation to the measure-theoretic metric invariants extent, mean distance and symmetry ratio. The symmetry ratio is a new invariant, taking values in $(0,1]$, derived from the first two, which have been studied previously in, e.g., [HKM] and [K1]. In the paper [K1 a conjecture was put forward, which can be formulated as: $a$ compact Riemannian manifold with symmetry ratio 1 is a round sphere.

It turns out, as we shall see, that a quite general compact metric space of symmetry ratio 1 is also of negative type, and thus identifying Riemannian manifolds of negative type is a first step towards resolving the conjecture.

The main result in this paper is given in Corollary 2.6, stating that a compact, globally symmetric Riemannian manifold of negative type is a round sphere. This then settles the conjecture in the class of Riemannian symmetric spaces.

Whether negative type itself characterizes the round spheres among compact Riemannian manifolds is to the author's knowledge still unsettled. Indeed the role of negative type in Riemannian geometry is still somewhat mysterious. However, the relation of negative type to the metric invariants we shall discuss below is, in the author's opinion, a good justification for studying the concept.

1.1. Background and notation. For background on measures and integration we refer to $[\mathrm{GP}$, while for background on Riemannian geometry we refer to $\mathrm{C}$. Here we will give a brief recap of the essential definitions from measure theory, since these might be unfamiliar to some geometers.

Received by the editors August 24, 2006.

2000 Mathematics Subject Classification. Primary 51K99, 53C35, 31C99.

The author was supported by the Danish Research Agency.

(C)2007 American Mathematical Society Reverts to public domain 28 years from publication 
Let $(X, d)$ denote a compact metric space. To avoid trivialities we always assume that $X$ contains at least two points.

The set of distributions, signed measures or Radon charges (as they are called in GP] is the $\mathbb{R}$-span of all Radon measures on $X$. We shall use the notation $\mathfrak{M}(X)$ to denote the set of Radon charges.

The norm or total absolute mass of $\mu \in \mathfrak{M}(X)$ is $\|\mu\|=|\mu|(X)$, where $|\mu|$ denotes the measure $\mu^{+}+\mu^{-}$, and $\mu^{+}, \mu^{-}$is the decomposition of $\mu$ into its positive and negative parts. With this norm $\mathfrak{M}(X)$ is a Banach space isometrically isomorphic to $C(X)^{*}$, the dual of the space of continuous functions $C(X)$ with the uniform norm, GP, Proposition 6.5.9].

$\mathfrak{M}(X)$ is equipped with the $w^{*}$-topology (the weak topology):

$$
\mu_{n} \rightarrow \mu \text { iff } \int_{X} f \mu_{n} \rightarrow \int_{X} f \mu \text { for all } f \in C(X) .
$$

The subset of $\mathfrak{M}(X)$ consisting of normalized, positive measures, i.e. $\mu=|\mu|$, will be called probability measures:

$$
\mathcal{P}(X):=\{\mu \in \mathfrak{M}(X)|\mu(X)=1, \mu=| \mu \mid\} .
$$

The support of a distribution $\mu \in \mathfrak{M}(X)$ is the minimal closed subset $Y \subseteq X$ such that $|\mu|(X \backslash Y)=0$.

Given two distributions $\mu, \nu \in \mathfrak{M}(X)$,

$$
\mu \otimes \nu=\mu^{+} \otimes \nu^{+}-\mu^{+} \otimes \nu^{-}-\mu^{-} \otimes \nu^{+}+\mu^{-} \otimes \nu^{-}
$$

denotes the product distribution on $X \times X$. We have a symmetric, bilinear form on $\mathfrak{M}(X)$ associated to the metric $d$ :

Definition 1.1. Define a symmetric bilinear form $I: \mathfrak{M}(X) \times \mathfrak{M}(X) \rightarrow \mathbb{R}$ as

$$
I(\mu, \nu):=\int_{X \times X} d(\cdot, \cdot) \quad \mu \otimes \nu .
$$

The corresponding quadratic form is denoted by the shorthand $I(\mu):=I(\mu, \mu)$.

For a distribution $\mu \in \mathfrak{M}(X)$ we define the associated potential of $\mu$ as

$$
\mathbf{p}_{\mu}(p):=\int_{X} d(p, q) \mu(q)=\int_{X} d(p, q) \mu^{+}(q)-\int_{X} d(p, q) \mu^{-}(q) .
$$

Thus by Fubini's Theorem, cf. [GP], we have for $\mu, \nu \in \mathfrak{M}(X)$,

$$
I(\mu, \nu)=\int_{X} \mathbf{p}_{\mu} \nu=\int_{X} \mathbf{p}_{\nu} \mu .
$$

1.2. Metric invariants. To a general compact metric space $X$ we can associate the following metric invariant:

Definition 1.2. The extent of $X$ is defined as

$$
\operatorname{xt}(X):=\sup _{\mu \in \mathcal{P}(X)} I(\mu) .
$$

The extent is thus the maximal mean distance of $X$ with respect to probability measures on $X$. The concept was introduced and studied in [GM] by Grove and 
Markvorsen, who defined the quantity as:

$$
\begin{aligned}
& \operatorname{xt}(X):=\lim _{q \rightarrow \infty} \mathrm{xt}_{q}(X), \text { where } \\
& \operatorname{xt}_{q}(X):=\max \left\{\frac{1}{2}\left(\begin{array}{l}
q \\
2
\end{array}\right)^{-1} \sum_{i, j=1}^{q} d\left(p_{i}, p_{j}\right) \mid\left(p_{1}, \ldots, p_{q}\right) \in X^{q}\right\} .
\end{aligned}
$$

The equivalence of the definition used in [GM] and the measure-theoretic definition given above follows from the $w^{*}$-density of finitely supported measures; see e.g. the discussion in $\mathrm{K} 2$.

Definition 1.3. A probability measure $\mu \in \mathcal{P}(X)$ on a compact metric space $X$ is called a normalized volume measure if it has support everywhere:

$$
\mu(B(p, r))>0
$$

for all $p \in X$ and all $r>0$. Here $B(p, r):=\{q \in X \mid d(p, q)<r\}$ is the open ball of radius $r$, centered at $p$.

The mean distance of a compact metric space $X$ with a fixed normalized volume measure $\mu_{0}$ is defined to be

$$
\operatorname{md}(X)=I\left(\mu_{0}\right) .
$$

We will call the potential associated to $\mu_{0}$ the mean distance function and use the notation: $\operatorname{md}(p):=\mathbf{p}_{\mu_{0}}(p)$.

In general we are interested in volume measures that are naturally associated to the metric, e.g. Hausdorff measures. For a Riemannian manifold $M$ we will always take the fixed normalized volume measure to be the normalized Riemannian measure:

$$
\mu_{0}=\frac{1}{V} \operatorname{vol}, \quad V:=\operatorname{vol}(M) .
$$

We can then define yet another metric invariant, which we will call the symmetry ratio:

Definition 1.4. For a compact metric space with a fixed volume measure $\mu_{0}$ define

$$
\operatorname{sr}(X)=\frac{\operatorname{md}(X)}{\operatorname{xt}(X)} \in(0,1]
$$

The idea behind the definition is that the comparison between md and $\mathrm{xt}$ in a given class of metric spaces should give a measure of how symmetric a space is, and that, at least in certain classes of spaces, we can only have $\operatorname{sr}(X)=1$ if the space is maximally symmetric (see however Remark 1.8 below). The following was conjectured in [K1]:

Conjecture. A compact Riemannian manifold of maximal mean distance $\operatorname{md}(M)$ $=\mathrm{xt}(M)$ is a round sphere $\mathbb{S}_{\kappa}^{n}$.

Here the round sphere of dimension $n$ and curvature $\kappa, \mathbb{S}_{\kappa}^{n}$, is the standard simply connected space form of constant curvature $\kappa>0$ for $n \geq 2$, while for $n=1, \kappa>0$, $\mathbb{S}_{\kappa}^{1}$ is a circle of length $2 \pi / \sqrt{\kappa}$. It is not difficult to verify that a round sphere has symmetry ratio 1 ; cf. [K1].

We will establish the conjecture in the class of Riemannian symmetric spaces below. 
Example 1.5. We will consider $\operatorname{sr}\left(\mathbb{R} P^{2}\right)$ to give an example of a symmetric space with symmetry ratio less than 1 . In $\mathbb{R} P^{2}$ there is, up to symmetry, one configuration $X$ of 3 points having mutual distances $\frac{\pi}{2}$. These points are the vertices of a triangle with 3 right angles. The extent of such a configuration of points is equal to the mean distance, which is

$$
\mathrm{xt}(X)=\operatorname{md}(X)=\frac{2}{3} \frac{\pi}{2}=\frac{\pi}{3} .
$$

This follows since $X$ has strictly negative type, and the extent is thus uniquely realized; cf. [HKM]. Thus $\operatorname{xt}\left(\mathbb{R} P^{2}\right) \geq \operatorname{xt}(X)=\frac{\pi}{3}$ (in fact we claim, that we have equality here). The same construction works in higher dimensions to show that $\mathrm{xt}\left(\mathbb{R} P^{n}\right) \geq \frac{n}{n+1} \frac{\pi}{2}$.

The length of the boundary of a ball of radius $r<\frac{\pi}{2}$ on $\mathbb{R} P^{2}$ is the same as for the 2-sphere, length $(S(p, r))=2 \pi \sin (r)$. So using polar coordinates to calculate the mean distance function, which is constant, at a point $p$ we get

$$
\operatorname{md}(p)=\frac{1}{\operatorname{vol}\left(\mathbb{R} P^{2}\right)} \int_{0}^{\frac{\pi}{2}} r 2 \pi \sin (r) d r=\int_{0}^{\frac{\pi}{2}} r \sin (r) d r=1 .
$$

So we have:

$$
\operatorname{sr}\left(\mathbb{R} P^{2}\right) \leq \frac{3}{\pi} .
$$

We claim that one can verify, with some work, that $\operatorname{sr}\left(\mathbb{R} P^{n}\right) \rightarrow 1$ as $n \rightarrow \infty$.

We will now introduce the concept of negative type:

Definition 1.6. A compact metric space $X$ is said to be of negative type if $I(\mu) \leq 0$ for every $\mu \in \mathfrak{M}(X)$ with vanishing total mass, $\mu(X)=0$.

Negative type has been studied in many different contexts; see [HKM] or DL] for more details. Usually the concept is defined via finite subsets, by saying that $X$ is of negative type if for any finite subset $\left\{p_{1}, \ldots, p_{n}\right\} \subseteq X$ and any associated weight vector $x \in \mathbb{R}^{n}$ with $\sum_{i=1}^{n} x_{i}=0$ we have

$$
\sum_{i, j=1}^{n} x_{i} x_{j} d\left(p_{i}, p_{j}\right) \leq 0 .
$$

The equivalence of the measure-theoretic definition given above and the one using finite subsets follows, as for the extent, by $w^{*}$-density in $\mathfrak{M}(X)$ of finitely supported distributions.

Apart from the problem of classifying Riemannian manifolds of negative type, the main reason for introducing negative type here is the following:

Lemma 1.7. Let $X$ be a compact metric space with a normalized volume measure $\mu_{0}$. Then $X$ has maximal mean distance $\operatorname{md}(X)=\operatorname{xt}(X)$, i.e. $\operatorname{sr}(X)=1$, iff $\operatorname{md}(p)=\operatorname{md}(X) \forall p \in X$ and $X$ is of negative type.

Proof. For $\mu, \nu \in \mathfrak{M}(X)$, we have

$$
I(\mu+\nu)=I(\mu)+2 I(\mu, \nu)+I(\nu)=I(\mu)+2 \int_{X} \mathbf{p}_{\mu} \nu+I(\nu) .
$$

Assume that $\operatorname{md}(X)=x t(X)$. By [B, Theorem 2] (or Theorem 13, p. 89 in [K2]), we have for a measure $\mu \in \mathcal{P}(X)$ realizing $\operatorname{xt}(X)$ that $\mathbf{p}_{\mu}(p)=\operatorname{xt}(X)$ for all $p$ in the support of $\mu$. Thus since $\mu_{0}$ has support everywhere, the mean distance function 
must be constantly equal to $\operatorname{xt}(X)$. Secondly, if $X$ is not of negative type, then since measures of the type $f \mu_{0}, f \in L^{\infty}\left(X, \mu_{0}\right)$ are weakly dense in $\mathfrak{M}(X)$ (see Lemma 1.9), we can find an essentially bounded function such that $\int f \mu_{0}=0$ and $I\left(f \mu_{0}\right)>0$ (using that the map $\mu \mapsto I(\mu)$ is $w^{*}$-continuous; cf. [K2 ).

Possibly by scaling, we may also assume that $\nu_{0}:=(1+f) \mu_{0} \in \mathcal{P}(X)$. But then by (1.5),

$$
I\left(\nu_{0}\right)=I\left(\mu_{0}+f \mu_{0}\right)=I\left(\mu_{0}\right)+I\left(f \mu_{0}\right)>I\left(\mu_{0}\right)=\operatorname{xt}(X),
$$

since the term $2 \int_{X}$ md $f \mu_{0}$ vanishes due to constancy of the mean distance function. Hence $X$ must be of negative type.

On the other hand, if $\operatorname{md}(p)$ is constant and $X$ is of negative type, then by the variational formula (1.5), $I(\mu) \leq I\left(\mu_{0}\right)=\operatorname{md}(M)$, for all $\mu \in \mathcal{P}(X)$ by inserting $\nu=\mu_{0}-\mu$.

Remark 1.8. The lemma above gives a procedure for constructing metric spaces with maximal mean distance: Given a metric space $X$ of negative type, find a compact subset $Y$ such that the mean distance function is constant on $Y$. In this way one can construct infinitely many disconnected, nonhomogeneous subsets of Euclidean spaces having symmetry ratio 1. Thus for the symmetry ratio to give a "good" measure of the homogeneity of a space, one should at least restrict to a class of connected spaces.

On the other hand, the lemma also shows that a homogeneous space equipped with an invariant measure, which then obviously has constant mean distance function, has symmetry ratio 1 iff the space is of negative type. Thus if the symmetry ratio conjecture is true, negative type should characterize the round spheres among compact homogeneous manifolds. The more general question whether negative type alone characterizes the round spheres among all compact Riemannian manifolds is to the author's knowledge still open.

Lemma 1.9. For a compact metric space $X$ with a fixed normalized volume measure $\mu_{0}$, the subspace $\left\{L^{\infty}\left(X, \mu_{0}\right) \mu_{0}\right\} \subseteq \mathfrak{M}(X)$ is weakly dense.

Proof. It is easy to check that $\frac{1}{\mu_{0}(B(p, r))} \mathbf{1}_{B(p, r)} \rightarrow \delta_{p}$ as $r \rightarrow 0$, where $\mathbf{1}_{B(p, r)}$ is the indicator function on the ball $B(p, r)$ and $\delta_{p}$ is the Dirac point measure supported at $p$. Then the result follows since atomic distributions are weakly dense, GP, 2.5.8].

One can easily extend the lemma above to show that $\left\{C(X) \mu_{0}\right\}$ is $w^{*}$-dense in $\mathfrak{M}(X)$, for example via weak density of $C(X)$ in $L^{\infty}\left(X, \mu_{0}\right)$; cf. [GP, 6.5.12].

\section{Riemannian MANifoldS OF NEGATive TYPe}

Refer to $\mathrm{C}$ for background on Riemannian geometry and to $[\mathrm{H}]$ for Riemannian symmetric spaces. Here we will recap some essential notions.

If $\gamma: \mathbb{R} \rightarrow M$ is a unit speed geodesic in a Riemannian manifold $M$, we say that $q=\gamma(s), s>0$, is a cut point of $p=\gamma(0)$ along $\gamma$ if $d(p, \gamma(t))=t$ holds for $t \in[0, s]$ but not for any $t>s$. The cut locus of $p \in M, C(p)$, is the set of all cut points of $p$.

We say that a simply closed, unit speed geodesic, $\gamma$, of period $2 L$ is a circle if $\gamma$ is minimizing on any subarc of length $L$, i.e. $d(\gamma(s), \gamma(s+L))=L$ for any $s \in \mathbb{R}$. Then clearly $\gamma(s+L) \in C(\gamma(s))$ for any $s \in \mathbb{R}$. 
If the cut locus of some point $p \in M^{n}$ consists of only one point, $C(p)=\{q\}$, then $M$ is called a twisted sphere and is homeomorphic, but not necessarily diffeomorphic, to a standard sphere $\mathbb{S}^{n}$; see $[\mathrm{G}$.

Theorem 2.1. If a compact Riemannian manifold $M^{n}$ is of negative type and contains a circle, then the cut locus of any point along the circle consists only of the antipodal point on the circle. Hence $M$ is a twisted sphere.

Proof. We may obviously assume that $\operatorname{dim} M \geq 2$, since any compact 1-dimensional manifold is a circle $\mathbb{S}_{\kappa}^{1}$. Let $\gamma: \mathbb{R} \rightarrow M$ be the circle and put

$$
\mu=\delta_{\gamma(0)}+\delta_{\gamma(L)}-\delta_{\gamma(s)}-\delta_{\gamma(s+L)},
$$

where $2 L$ is the period of $\gamma, s \in(0, L)$ and $\delta_{p}$ is the Dirac point measure with support at $p$. Thus $\mu$ consists of two positive masses placed at antipodal points $p_{1}=\gamma(0), p_{2}=\gamma(L)$ of $\gamma$ and two negative masses at (another) pair of antipodal points $q_{1}=\gamma(s), q_{2}=\gamma(s+L)$. One easily verifies that such a configuration realizes $I(\mu)=0$. Thus the potential $\mathbf{p}_{\mu}$ should be constantly zero if $M$ is of negative type; see Lemma 2.3 .

Clearly $p_{2}$ is on the cut locus of $p_{1}$, but not on the cut locus of $q_{1}$ and $q_{2}$. Let $\omega$ be a smooth unit speed curve with $\omega(0)=p_{2}$. Then by the first variational formula (cf. [C] or Lemma 2.2):

$$
\frac{d}{d t}_{\mid t=0}\left(d\left(\omega(t), q_{1}\right)+d\left(\omega(t), q_{2}\right)\right)=0,
$$

since $q_{1}$ and $q_{2}$ have opposite directions to $p_{2}$. But $\frac{d}{d t}{ }_{t=0} d\left(\omega(t), p_{2}\right)=\left\|\omega^{\prime}(0)\right\|=1$ (understood as a directional derivative). Thus in order for $\mathbf{p}_{\mu}$ to be constant we must have for the directional derivative of the distance to $p_{1}:\left.\frac{d}{d t}\right|_{t=0} d\left(\omega(t), p_{1}\right)=-1$ for any such $\omega$, i.e. any direction at $p_{2}$ must be a minimal direction to $p_{1}$; cf. Lemma 2.2 below. But this means that $C\left(p_{1}\right)=\left\{p_{2}\right\}$ : A geodesic connecting $p_{2}$ to another cut point $q$ could be continued to a minimal geodesic connecting $p_{2}$ and $p_{1}$, which is clearly impossible. The same argument goes for any pair of starting points on the circle.

We state the following well-known result, which can be proven by first variation of arc length methods; cf. [C]:

Lemma 2.2. The distance functions $f(q):=d(p, q)$ on a Riemannian manifold $M$ are directionally differentiable at any point $q \in M$. For any unit speed curve $\omega$ with $\omega(0)=q$ we have for the directional derivative with respect to $\omega^{\prime}: \omega^{\prime} f:=$ $\left.\frac{d}{d t}\right|_{t=0} f(\omega(t))=-\cos (\alpha)$, where $\alpha$ is the infimum of angles between $\omega^{\prime}$ and the initial directions of minimal geodesics to $p$.

Lemma 2.3. If $X$ is of negative type and $I(\mu)=0$ for some $\mu \in \mathfrak{M}(X)$ with $\mu(X)=0$, then the potential $\mathbf{p}_{\mu}$ is constant.

Proof. Consider two points $p, q \in X$ and put $\nu=\delta_{p}-\delta_{q}$, the sum of two Dirac point measures. Then $\mu+\epsilon \nu$ has vanishing mass for $\epsilon \in \mathbb{R}$ and

$$
I(\mu+\epsilon \nu)=I(\mu)+2 \epsilon \int_{X} \mathbf{p}_{\mu} \nu+\epsilon^{2} I(\nu)=2 \epsilon\left(\mathbf{p}_{\mu}(p)-\mathbf{p}_{\mu}(q)\right)-2 \epsilon^{2} d(p, q) \leq 0 .
$$

By choosing $\epsilon$ small enough, we see that we must have $\mathbf{p}_{\mu}(p)=\mathbf{p}_{\mu}(q)$ in order to fulfill the inequality. 
A Riemannian manifold, where the cut locus of any point $p$ consists of exactly one point $Q(p)$ at a fixed distance $d$ common to all $p$, is isometric to a round sphere; this follows from the Blaschke Conjecture; cf. [C, p. 244].

On a homogeneous space, i.e. a Riemannian manifold where the isometry group acts transitively, all points have isometric cut loci. So if the cut locus of some point is a single point at some distance $d$, this is true everywhere, and the space must be a round sphere by the Blaschke Conjecture:

Corollary 2.4. A homogeneous compact Riemannian manifold of negative type containing a circle is isometric to a round sphere, $\mathbb{S}_{\kappa}^{n}$.

A Riemannian (globally) symmetric space, is a special type of homogeneous space where each point has an isometry, called the geodesic symmetry, $\sigma_{p}$ such that $\sigma_{p}(\gamma(t))=\sigma_{p}(\gamma(-t))$ for any geodesic $\gamma$ with $\gamma(0)=p$. See $[\mathbf{H}]$ for details.

Lemma 2.5. Any closed geodesic of minimal length on a compact Riemannian symmetric space is a circle.

Proof. Let $\gamma \subset M$ be a closed geodesic of minimal length $2 L$ among all closed geodesics on $M$. This set is nonempty; cf. $[\mathrm{H}$, Proposition 5.3, p. 356]. Also any self-intersecting geodesic on $M$ is simply closed (Proposition 5.2 in [H]). Given $t \in \mathbb{R}$ we will show that the arc $\gamma([t, t+L])$ is minimizing; this implies that $\gamma$ is a circle.

If the arc $\gamma([t, t+L])$ is not minimizing, there is a strictly shorter geodesic $\omega:\left[0, L^{\prime}\right] \rightarrow M$ connecting $\gamma(t)$ and $\gamma(t+L)$. Since $\gamma(t+L)$ is a fixpoint of the geodesic symmetry at $\gamma(t)$, we must have that $\omega\left(-L^{\prime}\right)=\gamma(t+L)$; thus $\omega$ is a simply closed geodesic of strictly shorter length, which is impossible.

Since any compact globally symmetric space has a circle and is a homogeneous space, we get our main result of this paper:

Corollary 2.6. A compact Riemannian globally symmetric space of negative type is a round sphere $\mathbb{S}_{\kappa}^{n}$.

\section{REFERENCES}

[B] G. Björck, Distributions of positive mass, Arkiv för Matematik, Bd. 3, nr. 21 (1956), 255-269. MR0078470 (17:1198b)

[C] I. Chavel, Riemannian Geometry - a modern introduction, Cambridge University Press, Cambridge, 1993.

[DL] M. Deza and M. Laurent, Geometry of Cuts and Metrics, Springer-Verlag, Berlin, 1997. MR 1460488 (98g:52001)

[G] K. Grove, Critical point theory for distance functions, Proc. Symp. Pure Math. 54, Part 3 (1993), 357-385. MR1216630 (94f:53065)

[GM] K. Grove and S. Markvorsen, New extremal problems for the Riemannian recognition program via Alexandrov geometry, J. Amer. Math. Soc. 8, no. 1 (1995), 1-28. MR1276824 (95j:53066)

$[\mathrm{H}]$ S. Helgason, Differential Geometry and Symmetric Spaces, Academic Press, New York, 1962. MR0145455 (26:2986)

[HKM] P. G. Hjorth, S. L. Kokkendorff and S. Markvorsen, Hyperbolic Spaces are of Strictly Negative Type, Proc. Amer. Math. Soc. 130 (2002), 175-181. MR1855636 (2002j:53031)

[K1] S. L. Kokkendorff, Characterizing the Sphere by Mean Distance, preprint, DMF-2006-07002, 2004.

[K2] S. L. Kokkendorff, Geometry \& Combinatorics, Ph.D. thesis, Department of Mathematics, Technical University of Denmark, 2002.

[GP] G. K. Pedersen, Analysis Now, Springer-Verlag, New York, 1989. MR971256 (90f:46001) 
Department of Mathematics, Technical University of Denmark, Building 303, 2800 KGS. Lyngby, Denmark

E-mail address: S.L.Kokkendorff@mat.dtu.dk 